\title{
Deep Neural Network Approach in EMG-Based Force Estimation for Human-Robot Interaction
}

\author{
Hang $\mathrm{Su}^{1}$, Member, IEEE, Wen $\mathrm{Qi}^{1}$, Member, IEEE, Hongbo $\mathrm{Gao}^{2}$, Member, IEEE, Zhijun $\mathrm{Li}^{2}$, Senior Member, \\ IEEE, Giancarlo Ferrigno ${ }^{2}$, Senior Member, IEEE, and Elena De Momi ${ }^{1}$, Senior Member, IEEE
}

\begin{abstract}
In the human-robot interaction, especially when hand contact appears directly on the robot arm, the dynamics of the human arm presents an essential component in human-robot interaction and object manipulation. Modeling and estimation of the human arm dynamics show great potential for achieving more natural and safer interaction. To enrich the dexterity and guarantee the accuracy of the manipulation, mapping the motor functionality of muscle using bio-signals becomes a popular topic. In this article, a novel algorithm was constructed using deep learning techniques to explore the potential model between surface electromyography (sEMG) signals of the human arm and interaction force for human-robot interaction. Its features were extracted by adopting the convolutional neural network (CNN) from the sEMG signals automatically without using prior knowledge of the biomechanical model. The experiments prove the lower error $(<0.4 N)$ of the designed regression by comparing it with other approaches, such as artificial neural network (ANN) and long short-term memory (LSTM). It should be also mentioned that the anti-noise ability is an important index to apply this technique in practical applications. Hence, we also add different Gaussian noises into the dataset to demonstrate the robustness against measurement noises by using the proposed model. Finally, it demonstrates the performance of the proposed algorithm using the Myo controller and KUKA LWR4+ robot.
\end{abstract}

Impact Statement-Predicting interaction force using surface electromyography (sEMG) is a popular technology in humanrobot interaction. It increases the safety and the intelligence of human-robot collaboration. The novel deep learning algorithm we constructed in this article to explore the potential model between surface electromyography (sEMG) signals of the human arm and interaction force for human-robot interaction. The convolutional neural network $(\mathrm{CNN})$ is implemented to extract features from the SEMG signals automatically without using prior knowledge of the biomechanical model. The experiments prove the lower error $(; 0.4 \mathrm{~N})$ of the designed regression by comparing it with other approaches. It should also be mentioned that the anti-noise ability is also considered to apply this technique for practical applications. This technique could offer an alternative way for predicting the interaction force of the human-robot interaction.

Index Terms-Force measurement, Human-robot interaction, Neural Networks, Surface electromyography

\footnotetext{
*This work was supported in part by the European Commission Horizon 2020 research and innovation program, under the project SMARTsurg, grant agreement No. 732515.

${ }^{1}$ Hang Su, Wen Qi, Giancarlo Ferrigno and Elena De Momi are with the Department of Electronics, Information and Bioengineering, Politecnico di Milano, 20133, Milan, Italy. (e-mail: hang.su, wen.qi, giancarlo.ferrigno, elena.demomi@polimi.it).

${ }^{2}$ Hongbo Gao and Zhijun Li are with Department of Automation, University of Science and Technology of China, China. (e-mail: zjli@ieee.org).
}

\section{INTRODUCTION}

$\mathbf{O}$ VER the past few years, the booming development of robot technology has been witnessed. As a core part of robotics, the human-robot interaction (HRI) has attracted widespread attention and has been applied in many fields, including industrial programming [1], household service [2], [3], disaster rescue [4], educational guidance [5], medical surgery [6]-[8]. Especially in the medical domain, HRI is playing a significant role. For instance, Fujji et al. [9] utilized a robotic arm with a rigid endoscope instead of a camera assistant to help surgeons realize real-time observation in laparoscopic surgery. By using a seven-degree-of-freedom redundant robot, a teleoperated minimally invasive surgery was completed through the direct interaction between human hand and robot arm [10], [11]. To guarantee the safe and natural interaction between the human and robot, it is necessary to model and estimate the dynamics of human arm.

However, the high flexibility of the arm determines that it is difficult to establish the model accurately, especially involving interactive torque and force tasks [12]. Also, the time-consuming matter has become a challenge. These factors can directly affect the safety and practicability of HRI, and even threaten personal safety under poor performance [13]. Hence, it requires strict procedures to match the state-ofthe-art robot with human's dexterous arm. Obviously, the force generated by muscle contraction affects the flexibility and efficiency of the arm [14], [15], which proves that the muscle force is an important indicator to control the robot arm smoothly. How to accurately and reproducibly estimate muscle force has become an important research target in biomechanics.

Nowadays, analyzing the motor functionality of muscle based on biological signals becomes an effective strategy. As one of the neurologic signals, the electromyography signal can directly reflect the level of muscle activity and movement intention, and it can be converted into motion commands to control myoelectric prostheses as well as robotic arms [16], [17]. Notably, the surface electromyography (sEMG) signals on human's skin can be collected easily. Therefore, sEMG signals are suitable for high-precision force estimation. In order to obtain a satisfactory interaction behaviour of the robot during HRI, the relationship between sEMG signals and the human's arm force (sEMG-force) is required to be explored.

There are many existing parametric algorithms used to establish force estimation models. In [18], Hashemi et al. combined angle-based sEMG amplitude calibration with par- 
allel cascade identification to achieve force estimation, and the experimental results showed that the proposed approach had lower estimation error rate in dynamic muscle contraction. The authors in [19] collected sEMG signals based on a high-density electrode grid, and used the nonnegative matrix factorization algorithm to process the raw signals. Although this proposal greatly improved the quality of predictive force and reduced the number of electrode, it was limited to estimate force under isometric contraction. By using statistical methods to extract and visualize sEMG recordings, it can estimate human force and motion well, and improve the sEMG-based robotic arm control system [20]. Linear parameter varying approach was used to explore the muscle-force relationship of human neuromusculoskeletal system, and it provided new possibilities for robot control [21]. The above methods can complete accurate modeling between arm muscle and force, but they are highly dependent on algorithm parameters. Also, the convergence time and structure complexity of algorithms need to be optimized. Furthermore, since sEMG is weak and nonstationary, noise interference affects the performance of the estimator adversely.

Considering that parametric algorithms have many limitations, it is important to propose more nonparametric methods to build muscle-force models. Due to the expansion of data, the improvement of algorithms and the enhancement of computing power, machine learning (ML) and deep learning (DL) have gradually been used to solve robot-related problems. To solve the randomness and volatility of raw sEMG signals, the authors in [22] incrementally construct knowledge library based on decision tree by hierarchical projection regression algorithm. This proposal can project the original data to a lower dimensional feature space to match the real-time relationship between sEMG signals and motion state. A sEMGbased support vector machine approach [23] was presented to predict joint compression force, and the result of comparative experiments showed that it was a favourable estimator with low bias and high efficiency. Artificial neural network (ANN) was also used to extract the features of raw EMG signals in the time and frequency domains [24]. On the other hand, an increasing number of DL algorithms were developed to build force estimators, such as long short-term memory (LSTM), convolutional neural network (CNN), CNN-LSTM [25]. Ameri et al. [26] employed the regression $\mathrm{CNN}$ algorithm for independent and simultaneous motion control, but the robustness of the model needed to be further strengthened. Similarly, the author [27] proposed a recurrent deep neural network to estimate muscle forces, and then the accuracy of force model was improved based on transfer learning strategy. These nonparametric models, such as ANN and DL, do not need to capture prior information from arm muscles, and they have stronger anti-interference ability than parametric algorithms. When defining the input and output of the model, the best adaptive parameters can be trained.

A novel deep convolutional neural network (DCNN) is proposed in this paper based on a nonlinear regression model to map the interaction force and the sEMG signals. By combining several convolutional network layers, the ReLU activation function, and the dropout layer, the designed DCNN-based model aims to enhance the accuracy, save predictive time, and reduce the impact of noises. The model is an adaptive method which does not need any prior information and can extract valuable features automatically. Comparison experiments of the practical robot application are performed to demonstrate the efficiency of the proposed approach. Three major novel contributions of this work are concluded as follows:

1) A model-free approach using neural network is proposed to model the sEMG-force.

2) A novel nonliner regression algorithm using DCNN is implemented with capacity of fast computation, high accuracy and noise robustness.

3) The effectiveness and accuracy of the proposed method are depicted with a real-time demonstration.

\section{Problem Statement}

The potential relationship between sEMG and force can be denoted with a nonlinear mapping relation, which can be modeled using the mentioned DCNN model. We assume the input eight dimensions sEMG signals $S^{8}$ and the output one dimension force magnitude $F^{1}$. It should be noticed that, to ease the model complexity, we are using the force magnitude $F^{1}=\sqrt{f_{x}^{2}+f_{y}^{2}+f_{z}^{2}}$ instead of the force vector which features with direction, where $f_{x}, f_{y}$ and $f_{z}$ are the 3 -axis forces around three axes in the workspace. Because there are many noises in the raw sEMG signals, which affect the accuracy of the model, we propose a series of signal processing algorithms to solve these problems. The processed sEMG signals $S^{*}$ can be adopted to build the DCNN model $\Phi$, namely $\hat{F}_{t}=\Phi\left(S_{t}^{*}, \theta\right)$. Furthermore, $\theta$ is the parameter set of DCNN algorithm and the predicted force is defined as $\hat{F}_{t}$ at time $t$. In particular, $S_{t}^{*}$ represents the feature vector which is extracted from the uncertain probability $p_{t}\left(S_{t}^{*}\right)$ at time $t$. The force $F_{t}$ will change over time according to the input sEMG signals.

The aim of establishing model $\hat{F}_{t}=\Phi\left(S_{t}^{*}, \theta\right)$ is to find the best parameter space $\theta$, which can be determined by computing the loss function:

$$
\theta=L\left(\hat{F}_{j}-F_{j}\right)=\underset{\theta}{\operatorname{argmin}} \sum_{j=1}^{t}\left(\hat{F}_{j}-F_{j}\right)^{2}
$$

In this article, the regression accuracy of the proposed model is validated using the Mean Square Error (MSE), which is defined as

$$
\varepsilon=\sum_{j=1}^{t}\left(\frac{\hat{F}-F}{j}\right)^{2}
$$

\section{Methodology}

1) Training Data Preparation: The $8 \mathrm{D}$ raw sEMG signal $S^{8}$ was collected from the Myo armband, which should be extended into a matrix. It has been well known that the convolutional network is more efficient using the Homogeneous matrix [28]. Hence, a new input map $S^{8 \times 3}$ is constructed as:

$$
S^{*}=\left[S ; S-\bar{S} ; \frac{S-\bar{S}}{\sigma(S)}\right] ;
$$

the average and variance of $S$ are determined by the $\bar{S}$ and $\sigma(S)$, respectively. 
2) DCNN Architecture: As it is discussed in Section I, many methods to build the relationship between sEMG signals and robot end force ignore to consider about the performance of noise robustness and fast computation. The DCNN method is the best approach to achieve the goals. Therefore, we design a novel regression architecture based on the CNN. Fig. 1 illustrates the basic structure of the proposed DCNN regression model.

The DCNN is composed of five deep convolutional segments which includes a regression layer and a full connection layer. The whole deep convolutional segments is composed of a batch normalization (BN) layer, a $2 \mathrm{D}$ convolution layer and rectified linear units (ReLU). The detailed feature of the developed neural network model framework can be concluded as:

- Inputs: the $8 \mathrm{D}$ sEMG matrix $\tilde{S}^{8 \times 3}$. In Fig. 1, the 'inputs' shows the procedure of signal processing using Eq. 3 .

- Deep Convolutional Modules: Five deep convolutional modules are implemented using the proposed DCNN algorithm. The first one, which can be defined as Conv.Module \#1, consists of a 2D CNN layer, the second BN layer and a ReLU activation function. Although the last four convolution modules (Conv.Module \#2 to Conv.Module \#5) have the same layers, but they are filtered as a vector. The convolution operations were conducted basing on five window size, 4, 8, 12, 16 and 16. The sizes of yielded feature maps are $7 \times 2,6 \times 1$, $5 \times 1,4 \times 1$, and $3 \times 1$.

The convolution operations were conducted basing on the window size $2 \times 2$. The BN layer is exploited to grant every layer of the CNN to be trained by itself unsupervised. The ReLU layer is designed to settle the vanishing gradient and exploding gradient problems.

- Output: The output layer is constructed with the regression layer of a fully-connected layer. Finally, the predicted forces will be output.

\section{HARDWARE SYSTEM}

Fig. 3 depicts an overview of the system description, which was implemented for the task of physical human-robot interaction. The corresponding involved device information are listed below:

- The EMG sensor used in this experiment is the Myo armband, which is able to transmit the raw EMG information over a Bluetooth Smart connection with 8 Channels (200 $\mathrm{Hz}$ ).

- A KUKA robot is placed on the table to implement the task with physical human-robot interaction.

- The force sensor used in this paper is a 6-axis torque sensor. It is adopted to measure the online force of the physical human-robot interaction.

The gravity force of the tool tip has been eliminated in our previous works [29]-[31]. Hence the force sensor only measures the hand interaction force. The robot is working on Cartesian impedance control mode with a desired Cartesian pose to allow human-robot interaction.
In order to achieve high-efficient signal processing in realtime, the hand force estimation system was designed basing on two independent computers which communicated by the UDP protocol, so that the signals of force sensor and Myo armband can be mixed with timestamps. The force measurement developed in this work is similar to our previous work, which is conducted in real-time basing on the first computer with an i7-9750H CPU (2.60GHz) and 16 GB RAM [30]. The EMG signal collection node which is designed basing on ROS $^{1}$ Kinetic under Ubuntu is executed in the second computer with i7- $9700 \mathrm{~K}(2.9 \mathrm{GHz})$ and $16 \mathrm{~GB}$ RAM.

\section{EXPERIMENTS AND DISCUSSION}

To demonstrate

In this paper, the efficiency of the novel proposed approach is demonstrated in a lab setup condition. We design three experiments to estimate the performance of the DCNN-based regression model for mapping the $8 \mathrm{D}$ sEMG signals to the force. The first one aims to prove the high accuracy of the proposed DCNN algorithm by comparing it with LSTM, multiple layers neural network (MNN) and single-layer neural network (SNN). Meanwhile, the online predictive time is the primary index to evaluate time efficiency. Lastly, we add two types of Gaussian noise into the sEMG signals to judge the noise robustness ability.

\section{A. Data Collection}

As it is described in Fig. 2, one participant wears the Myo armband and moves the end of the robot from top to down (up-down), also from the left side to right side (left-right), and from front to back (front-back). Fig. 4 show the three-hand movements in detail.

The subject was commanded to perform these three movements with a repetition of twenty times. The sampling frequency of the two devices is $200 \mathrm{~Hz}$. Finally, the 'up-down' dataset has 11828 samples, 'left-right' dataset has 10868 samples, and the 'front-back' dataset has 13932 samples, respectively. The collected $8 \mathrm{D}$ sEMG signals and force signals will be analyzed by MATLAB 2019a with a computer server (16.0 GB RAM, 2.80 GHz CPU and Intel(R) i7 Core).

\section{B. Force Estimation}

In this paper, the dataset was divided into two parts: the first $80 \%$ samples and the other $20 \%$ samples. The first were chosen for training the model, and the other for testing. By comparing the MSE values among DCNN, LSTM, SNN and MNN models (see Table I), the proposed DCNN model gets the lowest MSE to predict all of the three-hand movements. The SNN model has 30 nodes in the hidden layer, while MNN is set 20 nodes and 30 nodes in its two layers. The SNN gets the highest errors than the other approaches. The reason might be the chosen number of nodes is too less to regress the force. Hence, adopting two layers to build the MNN model can solve the under-fitting problem.

\footnotetext{
${ }^{1}$ Robot Operating System, http://www.ros.org/
} 


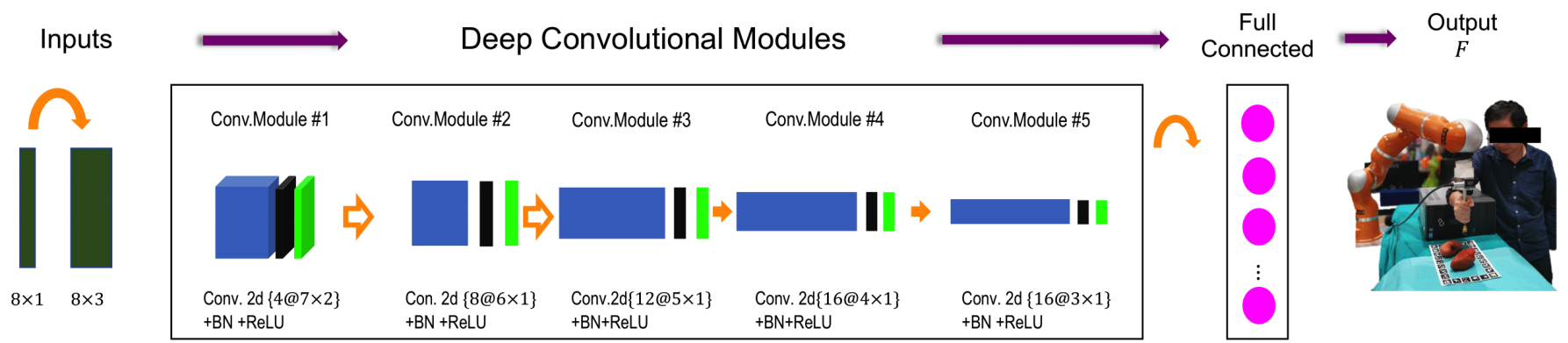

Fig. 1: The schematic diagram of proposed deep convolutional neural network (DCNN) structure.

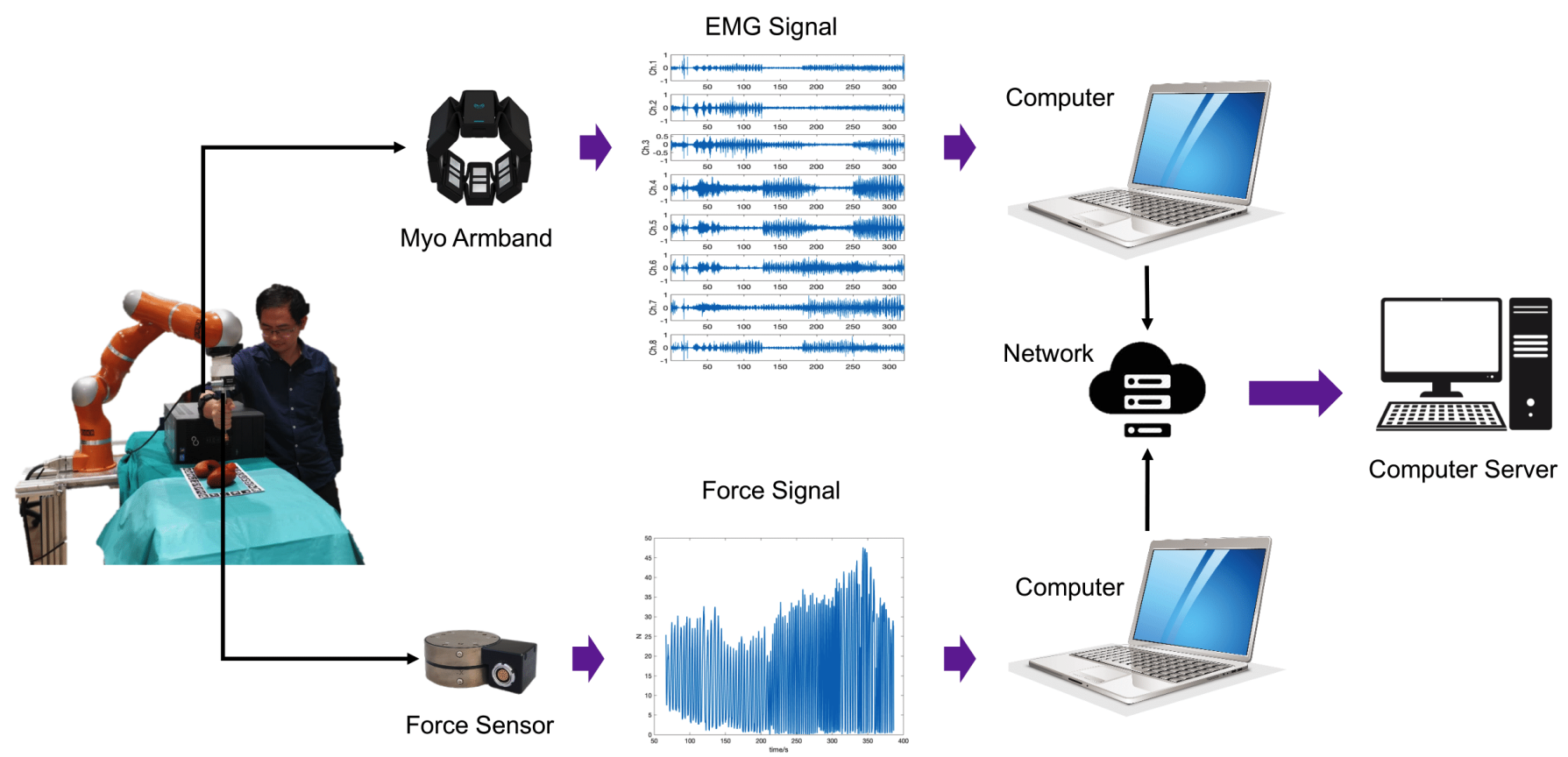

Fig. 2: The schematic diagram of EMG-based force estimation using force sensor and Myo armband.

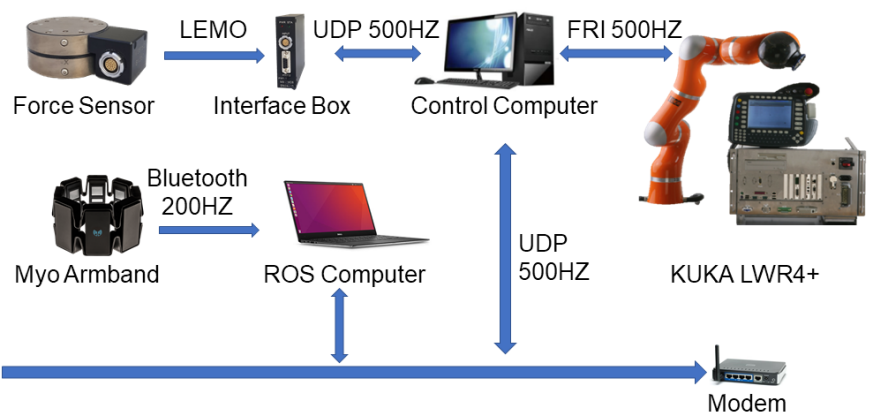

Fig. 3: Overview of the hardware system.

TABLE I: The comparison of MSE among DCNN, LSTM, SNN and MNN models.

\begin{tabular}{c|cccc}
\hline \multirow{2}{*}{ Hand Activity } & \multicolumn{4}{|c}{ Method } \\
\cline { 2 - 5 } & DCNN & LSTM & SNN & MNN \\
\hline Up-Down & 0.03 & 0.21 & 66.89 & 0.62 \\
Left-Right & 0.01 & 0.11 & 66.42 & 0.81 \\
Front-Back & 0.04 & 0.34 & 80.58 & 0.35 \\
\hline
\end{tabular}

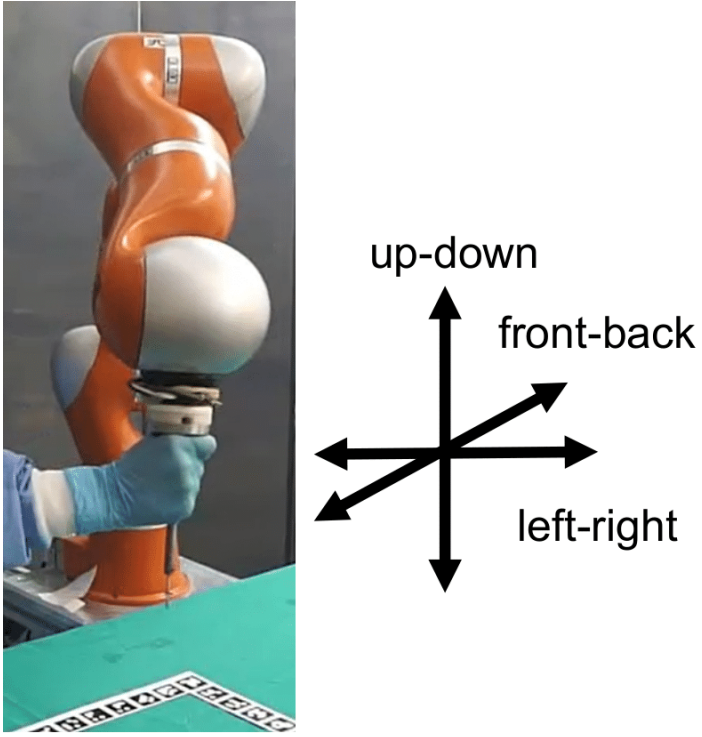

Fig. 4: The schematic diagram of the three hand movements.

The multiple convolutional layers not only can extract feature automatically but also save time to predict a result. 

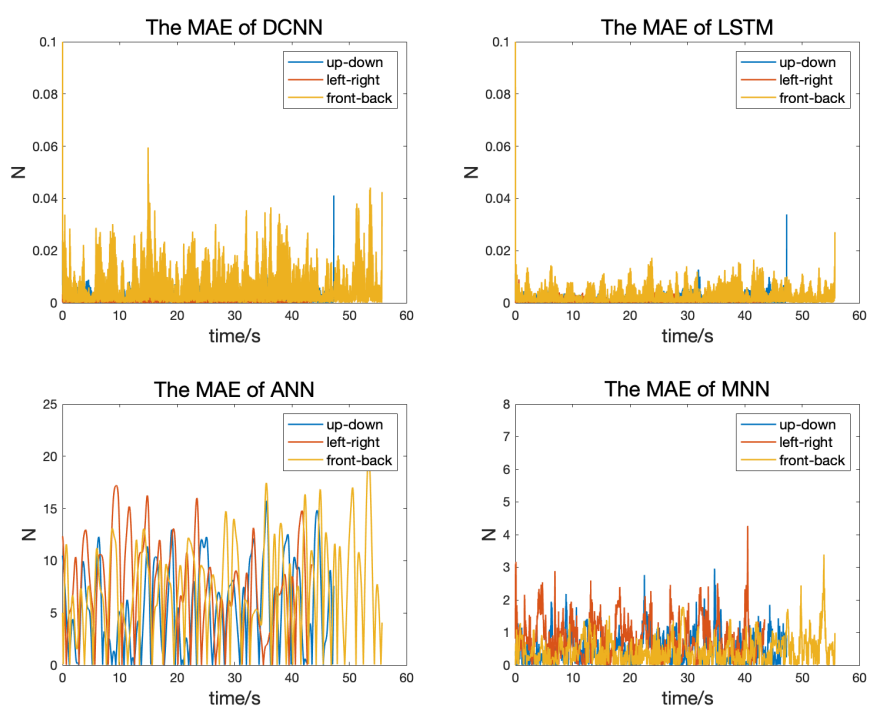

Fig. 5: The comparison mean absolute error (MAE) predicting on the training dataset among DCNN, LSTM, ANN and MNN models with the three different hand activities.
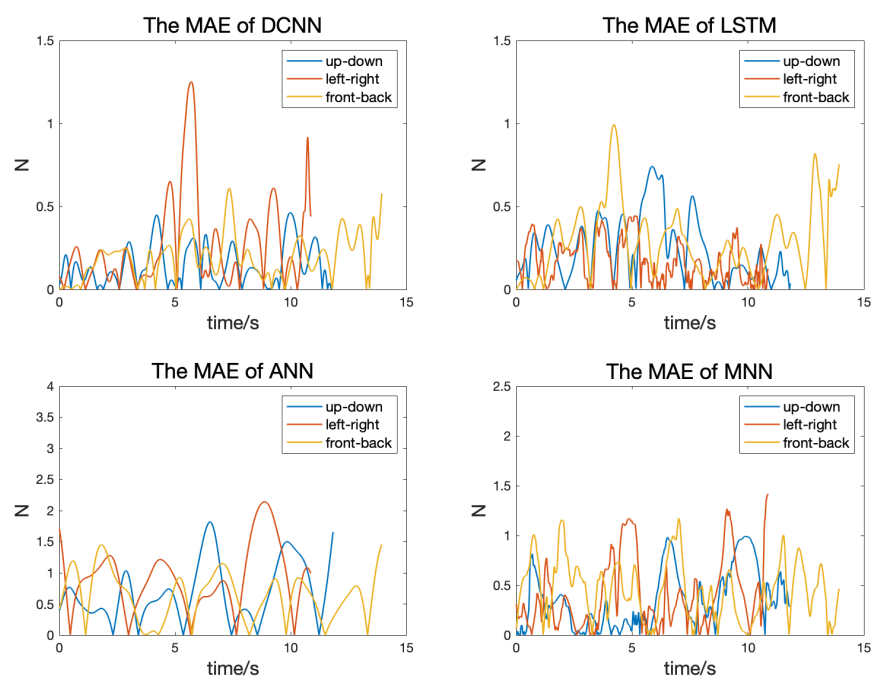

Fig. 6: The comparison mean absolute error (MAE) predicting on the testing dataset among DCNN, LSTM, ANN and MNN models with the three different hand activities.

TABLE II: The comparison of online predicting time (s) among different neural networks models, among DCNN, LSTM, SNN and MNN models.

\begin{tabular}{c|cccc}
\hline \multirow{2}{*}{ Hand Activity } & \multicolumn{4}{|c}{ Method } \\
\cline { 2 - 5 } & DCNN & LSTM & SNN & MNN \\
\hline Up-Down & 0.0007 & 0.0021 & 0.0063 & 0.0066 \\
Left-Right & 0.0008 & 0.0023 & 0.0060 & 0.0065 \\
Front-Back & 0.0008 & 0.0020 & 0.0062 & 0.0068 \\
\hline
\end{tabular}

Table II shows the online time by comparing these four models. It can be conclude that DCNN is an optimal method for predicting results in real-time. Specially, all of the ANNbased models requires plenty of time to predict a result, while the DCNN model only needs about 0.0008s.

We add Gaussian noises into the sEMG signals with two
TABLE III: The comparison of MSE with noise among the DCNN, LSTM, SNN and MNN models.

\begin{tabular}{c|cccc}
\hline \multirow{2}{*}{ Method } & \multirow{2}{*}{ SNR } & \multicolumn{3}{c}{ Hand Activity } \\
\cline { 2 - 5 } & & Up-Down & Left-Right & Front-Back \\
\hline \multirow{2}{*}{ DCNN } & $1 \mathrm{db}$ & 2.16 & 2.01 & 1.80 \\
\cline { 2 - 5 } & $5 \mathrm{db}$ & 12.27 & 13.38 & 12.94 \\
\hline \multirow{2}{*}{ LSTM } & $1 \mathrm{db}$ & 4.97 & 5.94 & 5.55 \\
\cline { 2 - 5 } & $5 \mathrm{db}$ & 27.96 & 30.00 & 29.44 \\
\hline \multirow{2}{*}{ SNN } & $1 \mathrm{db}$ & 10.01 & 9.73 & 9.83 \\
\cline { 2 - 5 } & $5 \mathrm{db}$ & 59.87 & 58.95 & 57.78 \\
\hline \multirow{2}{*}{ MNN } & $1 \mathrm{db}$ & 15.86 & 16.77 & 14.39 \\
\cline { 2 - 5 } & $5 \mathrm{db}$ & 66.38 & 68.86 & 69.05 \\
\hline
\end{tabular}

types of signal noise rate (SNR) (i.e., $1 \mathrm{db}$ and $5 \mathrm{db}$ ) to assess the accuracy of the trained DCNN model for its ability in noise robustness. Table III shows the comparison MSE among these methods, which proves that the DCNN is the best approach to remove the influence of noise.

The mean absolute error (MAE) of predicted forces on training and testing datasets are depicted in Fig. 5 and Fig. 6. The designed DCNN strategy obtains the lowest error than the other approaches. Fig. 7 shows the predictive curves of the three different training datasets. The SNN model cannot track the raw force curve, while the predicted curve of the DCNN model almost coincides with it.

\section{CONCLUSION}

In this paper, a nonlinear regression model is exploited using the DCNN technique to navigate the relation between the sEMG signals with the interaction force. It adopts the CNN to extract features from the sEMG signals automatically without using prior knowledge of the biomechanical model. The experiments prove the lower error $(<0.4 N)$ of the designed regression by comparing it with other approaches. Compared with traditional methodes such as SNN, MNN and LSTM, the proposed DCNN method improves the calculating performance and robustness to noise. Therefore, the DCNN method can improve the prediction accuracy of the dynamics force under noises. The feasibility of practical applications can also be ensured.

In the future, we will focus on more challenging problems in the sEMG-force control system, which can improve the accuracy of the DCNN regression method. Since this experiment is only performed on a fixed position, future works will involve position-free validation. Furthermore, the developed force estimation model could also be introduced to enhance the human-robot interaction.

\section{REFERENCES}

[1] S. Ong, A. Yew, N. Thanigaivel, and A. Nee, "Augmented realityassisted robot programming system for industrial applications," Robotics and Computer-Integrated Manufacturing, vol. 61, p. 101820, 2020.

[2] S. Cremer, F. Mirza, Y. Tuladhar, R. Alonzo, A. Hingeley, and D. O. Popa, "Investigation of human-robot interface performance in household environments," in Sensors for Next-Generation Robotics III, vol. 9859. International Society for Optics and Photonics, 2016, p. 985904.

[3] A. Yorozu and M. Takahashi, "Service robot using estimation of body direction based on gait for human-robot interaction," in International Conference on Intelligent Autonomous Systems. Springer, 2018, pp. 199-209. 


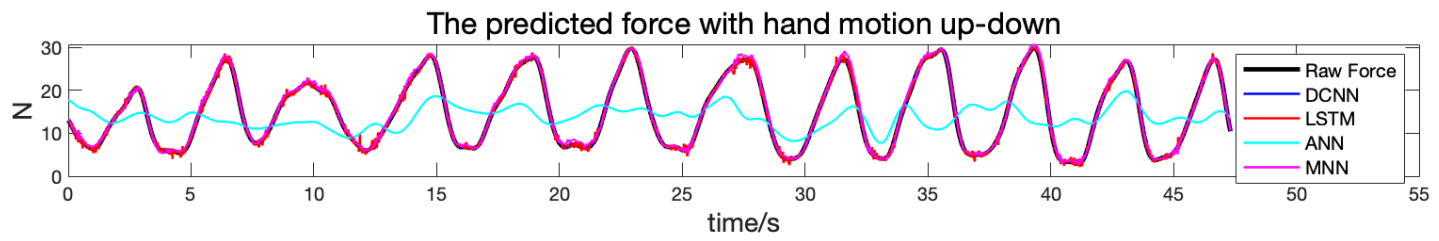

The predicted force with hand motion left-right

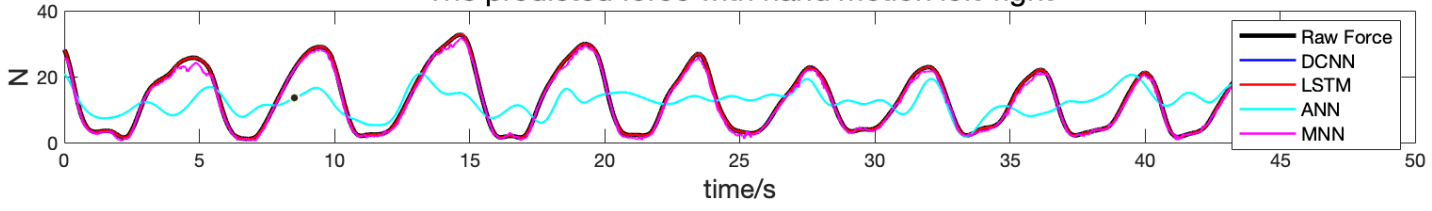

The predicted force with hand motion front-back

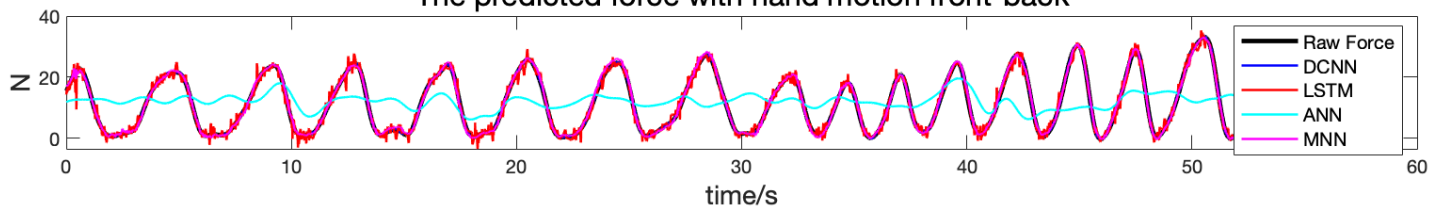

Fig. 7: The comparison force curves testing on training dataset among DCNN, LSTM, ANN and MNN models with the three different hand activities. The top graph is 'up-down', the middle one is 'left-right', and the bottom graph is 'front-back'.

[4] M. Lewis, K. Sycara, and I. Nourbakhsh, "Developing a testbed for studying human-robot interaction in urban search and rescue," in Proceedings of the 10th International Conference on Human Computer Interaction (HCII'03), 2019, pp. 270-274.

[5] J. Nasir, U. Norman, W. Johal, J. K. Olsen, S. Shahmoradi, and P. Dillenbourg, "Robot analytics: What do human-robot interaction traces tell us about learning?" in 2019 28th IEEE International Conference on Robot and Human Interactive Communication (RO-MAN). IEEE, 2019, pp. $1-7$.

[6] H. Su, S. Li, J. Manivannan, L. Bascetta, G. Ferrigno, and E. De Momi, "Manipulability optimization control of a serial redundant robot for robot-assisted minimally invasive surgery," in 2019 International Conference on Robotics and Automation (ICRA). IEEE, 2019, pp. 1323-1328.

[7] H. Su, S. Ertug Ovur, Z. Li, Y. Hu, K. Alois, J. Li, G. Ferrigno, and E. De Momi, "Internet of things (iot)-based collaborative control of a redundant manipulator for teleoperated minimally invasive surgeries," in 2020 International Conference on Robotics and Automation (ICRA). IEEE, 2020.

[8] A. E. Abdelaal, P. Mathur, and S. E. Salcudean, "Robotics in vivo: A perspective on human-robot interaction in surgical robotics," Annual Review of Control, Robotics, and Autonomous Systems, vol. 3, 2020.

[9] K. Fujii, G. Gras, A. Salerno, and G.-Z. Yang, "Gaze gesture based human robot interaction for laparoscopic surgery," Medical image analysis, vol. 44, pp. 196-214, 2018.

[10] H. Su, C. Yang, G. Ferrigno, and E. De Momi, "Improved humanrobot collaborative control of redundant robot for teleoperated minimally invasive surgery," IEEE Robotics and Automation Letters, vol. 4, no. 2, pp. 1447-1453, 2019

[11] Z. Li, B. Huang, Z. Ye, M. Deng, and C. Yang, "Physical humanrobot interaction of a robotic exoskeleton by admittance control," IEEE Transactions on Industrial Electronics, vol. 65, no. 12, pp. 9614-9624, 2018.

[12] J. Luo, C. Liu, and C. Yang, "Estimation of emg-based force using a neural-network-based approach," IEEE Access, vol. 7, pp. 64856$64865,2019$.

[13] E. Datteri, "Predicting the long-term effects of human-robot interaction: A reflection on responsibility in medical robotics," Science and engineering ethics, vol. 19, no. 1, pp. 139-160, 2013.

[14] L. Peternel, C. Fang, N. Tsagarakis, and A. Ajoudani, "Online human muscle force estimation for fatigue management in human-robot comanipulation," in 2018 IEEE/RSJ International Conference on Intelligent Robots and Systems (IROS). IEEE, 2018, pp. 1340-1346.

[15] S. Kim, J. Kim, M. Kim, S. Kim, and J. Park, "Grasping force estimation by semg signals and arm posture: tensor decomposition approach," Journal of Bionic Engineering, vol. 16, no. 3, pp. 455-467, 2019.

[16] A. Krasoulis, S. Vijayakumar, and K. Nazarpour, "Evaluation of regression methods for the continuous decoding of finger movement from surface emg and accelerometry," in 2015 7th International IEEE/EMBS Conference on Neural Engineering (NER). IEEE, 2015, pp. 631-634.

[17] M. Al Harrach, B. Afsharipour, S. Boudaoud, V. Carriou, F. Marin, and R. Merletti, "Extraction of the brachialis muscle activity using hd-semg technique and canonical correlation analysis," in 2016 38th Annual International Conference of the IEEE Engineering in Medicine and Biology Society (EMBC). IEEE, 2016, pp. 2378-2381.

[18] J. Hashemi, E. Morin, P. Mousavi, and K. Hashtrudi-Zaad, "Enhanced dynamic emg-force estimation through calibration and pci modeling," IEEE Transactions on Neural Systems and Rehabilitation Engineering, vol. 23, no. 1, pp. 41-50, 2014.

[19] C. Huang, X. Chen, S. Cao, B. Qiu, and X. Zhang, "An isometric muscle force estimation framework based on a high-density surface emg array and an nmf algorithm," Journal of Neural Engineering, vol. 14, no. 4, p. 046005, 2017.

[20] M. V. Liarokapis, P. K. Artemiadis, P. T. Katsiaris, K. J. Kyriakopoulos, and E. S. Manolakos, "Learning human reach-to-grasp strategies: Towards emg-based control of robotic arm-hand systems," in 2012 IEEE International Conference on Robotics and Automation. IEEE, 2012, pp. 2287-2292.

[21] M. Pesenti, Z. Alkhoury, M. Bednarczyk, H. Omran, and B. Bayle, "Linear parameter-varying identification of the emg-force relationship of the human arm," in 2019 28th IEEE International Conference on Robot and Human Interactive Communication (RO-MAN). IEEE, 2019, pp. 1-6.

[22] Y. Chen, X. Zhao, and J. Han, "Hierarchical projection regression for online estimation of elbow joint angle using emg signals," Neural Computing and Applications, vol. 23, no. 3-4, pp. 1129-1138, 2013.

[23] S. S. Li, C. C. Chu, and D. H. Chow, "Emg-based lumbosacral joint compression force prediction using a support vector machine," Medical engineering \& physics, vol. 74, pp. 115-120, 2019.

[24] G. Hajian, E. Morin, and A. Etemad, "Emg-based force estimation using artificial neural networks," CMBES Proceedings, vol. 42, 2019.

[25] L. Xu, X. Chen, S. Cao, X. Zhang, and X. Chen, "Feasibility study of advanced neural networks applied to semg-based force estimation," Sensors, vol. 18, no. 10, p. 3226, 2018.

[26] A. Ameri, M. A. Akhaee, E. Scheme, and K. Englehart, "Regression convolutional neural network for improved simultaneous emg control," Journal of neural engineering, vol. 16, no. 3, p. 036015, 2019.

[27] T. T. Dao, "From deep learning to transfer learning for the prediction of skeletal muscle forces," Medical \& biological engineering \& computing, vol. 57, no. 5, pp. 1049-1058, 2019.

[28] M. Tygert, J. Bruna, S. Chintala, Y. LeCun, S. Piantino, and A. Szlam, "A mathematical motivation for complex-valued convolutional networks," Neural computation, vol. 28, no. 5, pp. 815-825, 2016.

[29] H. Su, C. Yang, H. Mdeihly, A. Rizzo, G. Ferrigno, and E. De Momi, "Neural network enhanced robot tool identification and calibration for bilateral teleoperation," IEEE Access, vol. 7, pp. 122 041-122 051, 2019. 
[30] H. Su, W. Qi, C. Yang, J. Sandoval, G. Ferrigno, and E. De Momi "Deep neural network approach in robot tool dynamics identification for bilateral teleoperation," IEEE Robotics and Automation Letters, 2020.

[31] Z. Li, B. Huang, A. Ajoudani, C. Yang, C.-Y. Su, and A. Bicchi, "Asymmetric bimanual control of dual-arm exoskeletons for humancooperative manipulations," IEEE Transactions on Robotics, vol. 34, no. 1 , pp. 264-271, 2017.

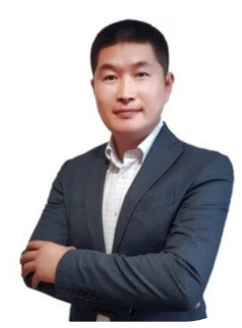

Hang Su (S'12-M'19) received the M.Sc. degree in control theory and control engineering in South China University of Technology, Guangzhou, China, in 2015 and the Ph.D. Degree in Bioengineering from Politecnico di Milano, Milano, Italy, in 2019. He participated in the EU funded project (SMARTsurg) in the field of Surgical Robotics. Dr. Hang $\mathrm{Su}$ is currently working in the Department of Electronics, Information and Bioengineering (DEIB) of Politecnico Di Milano. He is currently a Special Session Chair of IEEE International Conference on Advanced Robotics and Mechatronics (ICARM 2020). He also severs as the Associate Editor for the IEEE International Conference on Robotics and Automation (ICRA) and the IEEE/RSJ International Conference on Intelligent Robots and Systems (IROS), the IEEE International Conference on Robot and Human Interactive Communication (Ro-man) and the IEEE Internationa Conference on Advanced Robotics and Mechatronics (ICARM), and the Guest Editor for a couple of journals, like IEEE Robotics and Automation Letters, Complexity, Actuators, Mathematical Problems in Engineering, Sensors, Frontiers in Robotics and AI and Cognitive Computation and Systems, etc..

He has published over 50 papers in international conferences and journals and has been awarded RAS travel grant in ICRA 2019. His main research interests include control and instrumentation in medical robotics, human-robot interaction, surgical robotics, deep learning, bilateral teleoperation, etc..

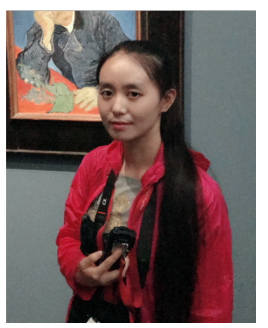

Wen Qi (S'19-M'20) received the M.Sc. degree in Control engineering from the South China University of Technology, Guangzhou, China, in 2015 and the Ph.D. Degree in Bioengineering from Politecnico di Milano, Milano, Italy, in 2020. She has published over 20 papers in international conferences and journals. Her first-authored paper was awarded the finalist of T J Tarn's Best Paper Award on Control Applications on IEEE WCICA 2014. She is serving as the Guest Editor for Cognitive Computation and Systems. Her main research interests include machine learning, deep learning and signal processing algorithms in wearable medical devices.

Hongbo Gao received the $\mathrm{Ph}$. D. degrees from Beihang University, Beijing, China, in 2016. He is currently an associate professor with the Department of Automation, School of Information Science and Technology, University of Science and Technology of China, Anhui Province, China, $\mathrm{He}$ is the author or coauthor of over 30 journal papers, and he is the co-holder of 6 patent applications. His current research interests include unmanned system platform and robotics, machine learning, decision support system, intelligent driving.

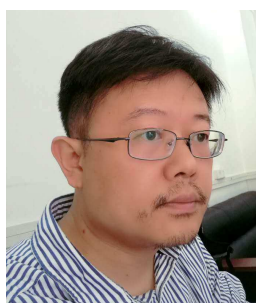

ZHIJUN LI (M'07-SM'09) received the Ph.D. degree in mechatronics, Shanghai Jiao Tong University, P. R. China, in 2002. From 2003 to 2005, he was a postdoctoral fellow in Department of Mechanical Engineering and Intelligent systems, The University of Electro-Communications, Tokyo, Japan. From 2005 to 2006, he was a research fellow in the Department of Electrical and Computer Engineering, National University of Singapore, and Nanyang Technological University, Singapore. Since 2012, he was a Professor in College of Automation Science and Engineering, South China university of Technology, Guangzhou, China. From 2017, he is a Professor in Department of Automation, University of Science and Technology, Hefei, China.

From 2016, he has been the Co-Chairs of IEEE SMC Technical Committee on Bio-mechatronics and Bio-robotics Systems $\left(B^{2} S\right)$, and IEEE-RAS Technical Committee on Neuro-Robotics Systems. He is serving as an Editor- at-large of Journal of Intelligent \& Robotic Systems, and Associate Editors of several IEEE Transactions. Dr. Li's current research interests include wearable robotics, tele-operation systems, nonlinear control, neural network optimization, etc.

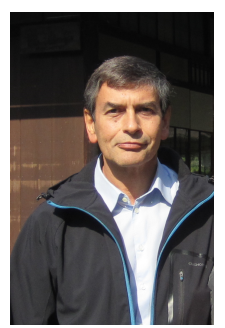

Giancarlo Ferrigno (M'15-SM'15) received the M.Sc. degree in electrical engineering and the Ph.D. degree in bioengineering from the Politecnico di Milano, Milan, Italy.

$\mathrm{He}$ is the Founder of the Neuroengineering and Medical Robotics Laboratory with the Department of Electronics, Information and Bioengineering, Politecnico di Milano, in 2008, and a Lecturer of Medical Robotics. He is a Full Professor with the Politecnico di Milano. He has been the European Coordinator of three FP7 EU projects on ICT. Two of them, ROBOCAST (STREP 2008-2010) and ACTIVE (Integrated project 2011-2015) are in the field of the Surgical Robotics. MUNDUS (STREP 2010-2013) is in the field of Assistive and Rehabilitative Robotics. He has co-authored 20 papers (ISI Web of Knowledge) in the robotic field from 2011 to 2014. Dr. Ferrigno is working in the JWG9 ISO standard group for Surgical Robots collateral standard and organized several workshops in the surgical robotics for the last three years.

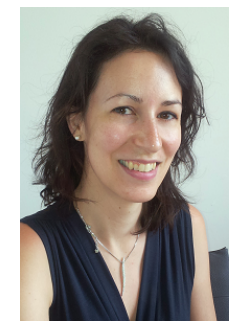

Elena De Momi (M'15-SM'18) received the M.Sc. and $\mathrm{Ph} . \mathrm{D}$. degrees in biomedical engineering from the Politecnico di Milano, Milan, Italy, in 2002 and 2006, respectively.

She is an Associate Professor with the Department of Electronics, Information and Bioengineering, Politecnico di Milano. She is co-founder of the Neuroengineering and Medical Robotics Laboratory, in 2008, being responsible of the Medical Robotics section. IEEE Senior Member, she is currently Associate Editor of the Journal of Medical Robotics Research, of the International Journal of Advanced Robotic Systems, Frontiers in Robotics and AI and Medical \& Biological Engineering \& Computing. From 2016 she has been an Associated Editor of IEEE ICRA, IROS and BioRob and she is currently Publication Co-Chair of ICRA 2019. She is responsible for the lab course in Medical Robotics and of the course on Clinical Technology Assessment of the MSc degree in Biom. Eng. at Politecnico di Milano and she serves in the board committee of the $\mathrm{PhD}$ course in Bioengineering. 